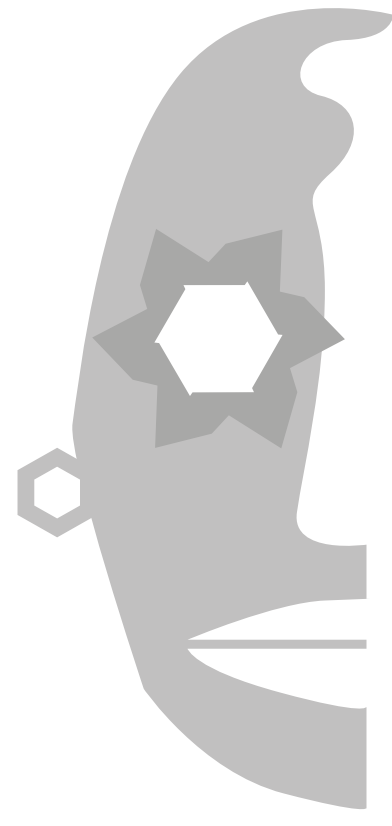

Kevin Pérez Méndez

Universidad de Costa Rica Costa Rica

\title{
Los bordes de la escritura: la crónica modernista de Arturo Ambrogi
}

\section{Resumen:}

Este artículo propone una exploración de los textos compilados en Marginales de la Vida (1963), título publicado por el salvadoreño Arturo Ambrogi en 1912, a partir de los vínculos que se establecen en ellos entre la influencia de la estética modernista y el oficio periodístico practicado por el autor. Los objetivos centrales son aproximarse a la producción literaria de Arturo Ambrogi mediante un análisis de las tensiones generadas al momento de ubicar parte de su escritura dentro de la literatura salvadoreña, centroamericana y latinoamericana de principios del siglo $\mathrm{XX}$, a la vez que se postula la posible existencia de un híbrido literario que rehuyó en gran medida de los puntos de vista seguidos por otros autores contemporáneos de la región durante la época en estudio.

Palabras clave: modernismo, crónica, Arturo Ambrogi, El Salvador

\section{Abstract:}

This article proposes an approach to a series of texts compiled in Marginales de la Vida (1963), a book published by Salvadorian writer Arturo Ambrogi in 1912, from the existing links on them between the modernist influence and the journalistic career developed by its author. The main objectives are to explore Arturo Ambrogi`s literary production through an analysis of the tensions generated when locating part of his writing in the Salvadorian, Central American and Latin American literature from the early XX century, and to postulate the possible presence of a literary hybrid that escaped from the visions followed by other contemporary writers of the region during the studied period.

Keywords: modernism, chronicle, Arturo Ambrogi, El Salvador 
El proceso de conformación de las primeras formas de literatura en las naciones latinoamericanas puede ser analizado a la luz de los acontecimientos que, de uno $\mathrm{u}$ otro modo, las llevaron en primeras instancias a convertirse en territorios independientes y autónomos. Sin duda alguna este hecho implica la aparición de esfuerzos o iniciativas, cuya principal pretensión es la de dotar a estos nuevos espacios de un aparato de símbolos con los cuales la sociedad se pueda identificar emocionalmente; un aparato que incluye, por supuesto, al discurso literario en tanto testimonio de las experiencias vividas. El siglo XIX se encuentra atravesado de principio a fin por esta clase de formulaciones nacionalistas o regionalistas, sin embargo, en el caso centroamericano, también es posible notar cómo este deseo por utilizar la literatura para registrar los cambios de un determinado lugar sigue plenamente vigente de cara al inicio del siglo XX, mediante la utilización de recursos que, dados las circunstancias de la época, representan una forma bastante particular de hacer literatura.

El presente artículo se sitúa temporalmente en las primeras dos décadas del siglo $\mathrm{XX}$, tomando como punto central el momento de publicación de Marginales de la Vida (1963), una compilación de textos breves escrita por el autor salvadoreño Arturo Ambrogi y publicado en su país por primera vez en 1912. La referencia temporal también sirve para enmarcar los dos espacios dentro de los cuales el texto desea ser estudiado: por un lado, la impronta tardía de las ideas modernistas promulgadas a finales del siglo XIX en el continente americano, y por el otro, el ascenso y el desarrollo que el género de la crónica tuvo en el periodo finisecular y que llegaría a consolidarse en la región durante el periodo inicial del siglo XX.

La tesis central de este análisis es estudiar el modo (o los modos) en el cual los textos de Arturo Ambrogi se acercan o se distancian de dichos polos; debido a esto, se planean en primer lugar algunas reflexiones acerca estas dos vertientes: la naturaleza estética-estilística fundamental del modernismo, y el carácter periodístico con el que algunos de los textos en Marginales de la Vida (1963) son enunciados. Así, se parte del supuesto según el cual los textos escogidos oscilan en algún lugar entre estas dos formas de escritura y, por lo tanto, vienen a representar un punto de encuentro entre el discurso literario y periodístico en la escritura de Ambrogi, la cual, dicho sea de paso, se encuentra enmarcada en el costumbrismo salvadoreño de principios de siglo.

De este modo, también se hace necesario aclarar que para efectos del análisis la selección de los textos se ha denominado y se denominará solo a partir de este término -texto-, ya que también se pretende observar la categorización que en algunos casos se le da a los mismos como crónicas, cuadros de costumbres o incluso relatos; un aspecto que además contribuye a complementar la tesis explicada acerca de la falta de claridad al momento de definir el lugar que ocupa la obra 
de Arturo Ambrogi dentro de la esfera literaria de Centroamérica. Finalmente, es pertinente señalar que el estudio se enfocará en cinco de los textos presentes en Marginales de la vida (1963), aunque esto no descarta la mención de otros títulos compilados en dicho libro.

A lo anterior se le suma la evidencia encontrada al repasar otros elementos que son directamente ajenos al texto por estudiar, pero que forman parte de su entorno e influyen en el acercamiento que se quiere alcanzar a través de este artículo. Ejemplos de esto son la figura misma del autor, y la ausencia de fuentes que desarrollen a fondo las consecuencias de movimientos como el modernismo en la nación salvadoreña. Sobre este tema, tal y como lo menciona Canales (1978), se debe tener en cuenta que la producción de Ambrogi se halla a medio camino entremezclada con distintos paradigmas literarios de la época, pues, al igual que otros autores latinoamericanos que escribieron a finales del siglo XIX e inicios del XX, su obra se abocó de manera abrupta sobre el crisol de las ideas románticas, naturalistas, modernistas e, incluso, simbolistas.

Es igualmente importante señalar, además, la intensa labor periodística practicado por Ambrogi a lo largo de toda su vida, la cual lo sitúa en la tradición como uno de los cronistas más prolíficos del istmo y de la nación salvadoreña. No obstante, y a pesar de dicho conocimiento general acerca de la figura del autor, resulta curioso observar la existencia de un vacío en cuanto a la clasificación de su obra, no solo respecto a la literatura de su país y de su época, sino también en lo que concierne al resto de la región latinoamericana. Esto se torna más notorio tomando en cuenta lo que argumentan autores como George J. Edberg (1965) sobre la importancia que tuvo la presencia de Ambrogi en América durante la consolidación de modernismo, en donde su obra se ubica muy de cerca con la de escritores como Rubén Darío, Leopoldo Lugones y José Ingenieros, con quienes el salvadoreño se relacionó directamente en distintos momentos.

Las líneas anteriores refuerzan la idea planteada al principio acerca de la posibilidad de comprender los modos de escritura en Marginales de la Vida (1963) a partir de la relación que dicha escritura establece con la esencia de la crónica y del modernismo. Ciertamente, no se trata de un problema completamente inexplorado; sin embargo, lo que llama la atención es conocer el periodo en el cual dicho diálogo pudo haberse producido pues, en el fondo, la construcción de un híbrido literario a inicios del siglo XX en la región podría interpretarse como el antecesor de otras formas igualmente entremezcladas, que fueron tomadas por autores y artistas en los años venideros y a lo largo del siglo anterior. 


\section{Construir lo moderno}

En primer lugar, el término modernismo necesitaría un espacio de estudio propio solamente para desarrollar las aproximaciones que se han hecho acerca de sus postulados y sus maneras de entenderse. Este artículo, no obstante, no tiene la intención de ocuparse de los límites o los modelos con los que la estética modernista se ha explicado sino, por el contrario, busca rastrear en dichos puntos de vista los factores que puedan señalarse como rasgos comunes, esto es, tendencias practicadas por los autores durante este período y que constituyen las pistas suficientes para determinar un influjo estilístico que se manifiesta en mayor o menor medida.

Al respecto, es de gran pertinencia lo señalado por Meyer-Minnemann (1987), quien establece una relación bastante obvia pero que frecuentemente es ignorada por quienes se dedican al estudio de la literatura. Dicho autor entabla una unión entre el surgimiento del modernismo en los años ochenta del siglo XIX y el proceso de modernización/industrialización que tomó lugar durante esta misma etapa histórica. Asimismo, Meyer-Minnemann (1987) utiliza la terminología de lo moderno para designar el surgimiento de un sistema de ideas novedoso, opuesto a los modelos antiguos, no contemporáneos y, por lo tanto, obsoletos. Quizás, entre sus afirmaciones, es importante rescatar la siguiente reflexión acerca de la innovación que el modernismo representó a pesar de haberse desarrollado en distintos ámbitos y en momentos temporalmente homogéneos pero separados:

(La escritura modernista) resultó novedosa gracias a la presentación de contenidos literarios que remitían a los aspectos social y técnicamente más avanzados de la vida coetánea, a hombres, objetos y/o circunstancias deliberadamente ajenas al medio ambiente del lector enfocado (...) Resultó novedosa, asimismo, la tentativa de los autores respectivos de crear un lenguaje que se prestara esos contenidos nuevos, un lenguaje que con la ayuda de medios expresivos se ajustara a ellos. (Meyer-Minnemann, 1987, p. 79)

De acuerdo con esto, la característica fundamental de modernismo surgido tanto en Europa como en Hispanoamérica reside en la incorporación de temas acordes a los avances que suponía el proceso de modernización en el ámbito cultural, social, económico y político. De igual modo, esta afirmación se enlaza con el carácter principalmente cosmopolita y exótico de la literatura perteneciente al periodo, en donde no pocas veces la ciudad ocupó el centro de atención de las más variadas impresiones.

En esta misma línea, también se puede comprender el refinamiento lingüístico mencionado por Meyer-Minnemann (1987) bajo la invención de nuevas maneras de usar el lenguaje para referirse a los objetos mediante un lente nuevo o al menos distinto. Dicho refinamiento conduce a su vez a lo que Acereda (2001) cita como 
la intención por modernizar, de cara al nuevo siglo, las ideas y la sensibilidad viciadas por el positivismo, al tiempo que se procuraba alcanzar la armonía entre la vida cotidiana y las formas artísticas más elevadas, con la cultura francesa como base y fundamento de prácticamente toda la inspiración.

De esta manera, las ideas de lo que debía ser lo moderno parecen encausarse y tomar forma en una serie de rasgos que, entre otras cosas, buscaban reescribir el presente y de algún modo preparar la consciencia de los individuos ante los cambios inminentes que las circunstancias finiseculares traían consigo. Esto, además, implicó la ocupación de espacios que hasta el momento no habían sido directamente tomados por quienes escribían, tal como sucede con la dimensión espiritual y el escapismo de la realidad y de la referencialidad que se retratan en muchos de los versos escritos durante el periodo en estudio.

En una buena parte de su ensayo, Meyer-Minnemann (1987) también rescata la naturaleza periférica del modernismo más consolidado. Su argumento se basa en el hecho de que, al formar parte de un territorio que no pertenecía al centro cultural del mundo ${ }^{1}$, las ideas modernistas tuvieron un amplio nicho de recepción tanto en España como en Latinoamérica, con el discurso poético como principal medio de expresión, característico de esta estética.

Lo anterior hace necesario enfocarse en el vínculo establecido por algunos de los autores estudiados para este artículo en relación con la realidad (o la idea de una realidad) de la cual los modernistas echaron mano para hacer literatura. Un estudio exhaustivo acerca de este aspecto requeriría un análisis separado acerca del espacio dentro del cual se desarrolló el modernismo a lo largo de las décadas. Por esta razón, conviene enfocarse de manera particular en los cambios sucedidos dentro del territorio latinoamericano $\mathrm{y}$, enfáticamente, en la nación salvadoreña de finales del siglo XIX e inicios del siglo XX, momento en el cual Arturo Ambrogi escribió los textos que se reúnen en el libro citado al inicio.

Al igual que en el resto del istmo, El Salvador gestó una buena parte de su discurso cultural a lo largo de las décadas del siglo XIX y como resultado de su independencia. La llegada de la industrialización supuso un nuevo cambio en la forma de ver el espacio y de pensar las ideas sobre lo que la sociedad debía o no alcanzar. En este sentido, es curioso observar cómo la modernización llevada a cabo en El Salvador, y que es retratada en parte de la obra de Ambrogi, no renuncia completamente al positivismo que caracterizaba las ideas modernistas en otras latitudes, pues, en cambio, adoptaba una buena parte del aparato intelectual promovido en la región a finales de siglo, tal y como lo afirma Bradford-Burns:

1 Representado en París. 
A new interest in science deflected judgments on progress, as well as progress itself, away from philosophical and moral matters toward material change. Some of the ideas concerning progress drawn from the Enlightenment, Darwin, Spencer, and other similar European sources blended into the form that Auguste Comte's positivism assumed in Central America during the last decade of the century. (Bradford-Burns, 1985, p. 61)

A lo anterior, Bradford-Burns (1985) agrega el surgimiento de una infraestructura liderada por intelectuales y académicos que se tomaron el deber de "urbanizar" las ideas y los tópicos que atravesaban el imaginario cultural de la nación. La aparición de academias y bibliotecas, así como el interés gubernamental por actualizar los métodos utilizados en la enseñanza, son solo algunos ejemplos del cambio que se proponía durante esta época. En cuanto a la literatura, la poesía parece haberse posicionado en un lugar central, primero con influencias evidentemente neoclásicas, y posteriormente con el modernismo "traído" al continente por autores como Rubén Darío y Francisco Gavidia.

Resulta importante notar que el estudio de Bradford-Burns, como el de otros autores, solo se limita a los ámbitos de lo poético y, si bien sus reflexiones son acertadas en lo relativo a los temas abordados por dicha forma literaria, no se señala en ninguna parte el papel que la prosa tuvo como discurso más directo y referencial. En principio, este aspecto podría interpretarse como una carencia de estudios sobre las narraciones escritas en la región durante esta época, sin embargo, las fuentes consultadas señalan que, por el contrario, la poca atención que se la ha dado a la prosa modernista se ve motivada porque dichas narraciones no se adaptan completamente a las ideas planteadas por la poesía o el modernismo en general. El caso de Ambrogi parece ejemplificar este hecho.

Un argumento similar es ya señalado por Edberg (1965), quien no solo reflexiona sobre el carácter periodístico de algunos textos escritos por Ambrogi ${ }^{2}$, sino también acerca de la escritura de dichos textos a partir de experiencias, viajes y acontecimientos de orden social registrados por el autor. La problematización propuesta por Edberg no es única, otros autores analizados también parecen encontrar una o varias dificultades al momento de hablar de la obra escrita por Arturo Ambrogi. Así, a la denominación de "artículos" también se le suma la hecha por Canales (1978), quien habla de "narraciones", o incluso la aseveración hecha por Uriarte en el prólogo del libro ${ }^{3}$, quien de hecho critica una buena parte de los textos por considerarlos "estampas" sin carácter social, ajenas a la realidad salvadoreña y escritas solo para leerse como un pasatiempo.

2 A los que incluso denomina "artículos".

3 Ambrogi, A. (1963). Marginales de la vida. $2^{\text {a }}$ ed. San Salvador: Ministerio de Educación. 
Pese a esto, es curioso observar que, a pesar de la relación que claramente se da entre la obra de Ambrogi y su ocupación periodística, ninguno de los autores estudiados vuelva sobre esta idea para elaborar una posible justificación sobre las dificultades de clasificar la empresa del autor salvadoreño en relación con otras formas discursivas cercanas a lo literario.

\section{Destruir los lindes}

El límite entre el periodismo y la literatura ha sido ampliamente discutido en estudios llevados a cabo por autores como Pérez-Grande (2008), quienes en general señalan la reticencia con la cual las vanguardias observan el estilo periodístico, al tiempo que apuntan la capacidad de este último de alcanzar recursos similares a los usados por quienes hacen ficción. Pérez-Grande, por ejemplo, sostiene que en realidad el oficio de la escritura se presenta de un modo similar en los dos casos, lo cual empuja y casi obliga a quienes escriben a echar mano de recursos provenientes de ambos polos: "En el plano de la escritura, tanto el escritor como el periodista, frente a la página en blanco, estará frente a un desafío ineludible. Para sobrevivir los dos, en este reto, tendrá que recurrir a todas las argucias posibles" (Pérez-Grande, 2008, p. 204).

De un modo similar, Reguillo (2000) cuestiona teóricamente la narración latinoamericana surgida en el último siglo y, junto a esta afirmación, la autora también propone a la crónica como un género que, de uno u otro modo, pone en tela de duda la escritura de los relatos según paradigmas sistemáticos. Este hecho además se une a lo que Reguillo (2000) define como el debilitamiento de las instituciones y su lugar de enunciación; ante tal acontecimiento, la experimentación de ciertos autores con la crónica se manifiesta como un lugar de narración que fragmenta el discurso y da cabida a espacios que antes se hallaban marginados o excluidos de la literatura. Su argumento más contundente es, quizás, la relación que establece entre la noción de la crónica como oposición al ideal literario modernista:

La crónica se re-coloca hoy frente al logos pretendido de la modernidad como discurso comprensivo, al oponerle a este, otra racionalidad, en tanto ella puede hacerse cargo de la inestabilidad de las disciplinas, de los géneros, de las fronteras que delimitan el discurso. (...) La crónica no debilita "lo real", lo fortalece, ya que su "apertura" posibilita la yuxtaposición de versiones y de anécdotas que acercan a territorio propio, es decir, (re)localizan el relato. (Reguillo, 2000, p. 24)

Reguilo (2000) también complementa lo anterior con algunas características formales acerca de la crónica latinoamericana escrita a inicios del siglo XX, tales como la linealidad, la crudeza tonal en lo que se cuenta y la ausencia de una autoría totalmente palpable dentro del texto. El cumplimiento o no de estas reglas 
formales en los textos de Ambrogi no posee mayor relevancia en este caso, ya que, en última instancia, una clasificación de esta naturaleza se opondría a la ambigüedad que se trata de problematizar aquí. Por el contrario, las afirmaciones de Reguillo parecen coincidir con lo que se señaló antes acerca de las narraciones modernistas y su dificultad para adaptarse de manera total a los esquemas estéticos que, al menos en la lírica, parecen ser más homogéneos.

Los argumentos de Reguillo (2000) también dejan ver que el surgimiento de la crónica se da más como un síntoma de la pérdida de vigencia de las instituciones modernas, lo cual, según la autora, dio paso a la desestabilización de los lugares de enunciación legítimos y a la inclusión de voces que antes habían sido marginadas, ya sea por el discurso cientificista o por la ortodoxia narrativa. Así, se rescatan la voz de personajes y lugares más cercanos a lo cotidiano, en donde la escritura ya no se centra en la legitimidad literaria de lo que se cuenta, si no en la visibilización de lo que antes era ignorado.

Al analizar algunos de los textos compilados en Marginales de la Vida (1963), es posible observar que, en principio, Ambrogi se apega estilísticamente a muchos de los tópicos explorados por la corriente modernista, pero, sin dejar dichos tópicos de lado, se vincula además con una esencia periodística en cuanto al carácter serio y contextual de lo que cuenta. Una muestra del apego estilístico mencionado se aprecia en el caso del texto que lleva por nombre Los primeros crisantemos (1963), en donde el autor utiliza la imagen de las flores para construir una suerte de oda al exotismo que se estilaba en una buena parte del Occidente respecto de la cultura oriental, en este caso, la japonesa:

El Japón, junto con sus lacas y bronces, nos impuso el crisantemo como una flor elegante; y el cuidado y la pasión de los floricultores franceses, hicieron de la plebeya de antes, de la antes despreciada, una flor opulenta y aristocrática; una flor de moda y de estética. (Ambrogi, 1963, p. 313)

Del mismo modo en el cual se describiría una obra de carácter pictórico y visual, Ambrogi se toma la tarea de ensalzar las cualidades que le otorgan a una planta la esencia lo suficientemente "elevada" como para ser el objeto de una parte de su escritura. Aunado a esto, las capas del entramado modernista que atraviesan lo que el autor cuenta se observan al ver cómo la flor, en tanto influencia y tendencia estética, pasa históricamente por las manos orientales y europeas antes de llegar al texto citado, donde la imagen es apropiada y escrita dentro de unas circunstancias (centroamericanas) bastante distintas a las que le dieron origen, sin que eso represente un obstáculo para que el autor le dedique varias páginas del libro. Ambrogi incluso parece ser consciente de que hay un peso contextual en las referencias que utiliza: "El crisantemo es el precursor del japonismo en Francia. Blacard, por puro negocio, sin saberlo, dio el primer paso. Luego los Goncourt 
con sus monografías de artistas amarillos y sus comentarios exóticos, impusieron la moda el arte fal" (Ambrogi, 1963, p. 317).

Además, si se piensa en lo explicado acerca de la aspiración europea de modernismo, es sencillo entender que la mención a la cultura francesa no es gratuita en este fragmento. Referencias similares, cargadas de estilismo y de musicalidad, se hallan dispersas en el libro y son rastreables desde el instante en el que el lector repasa los títulos y encabezados, tal y como sucede en L'Oncle Sarcey (1963), en donde el autor incluso inserta párrafos completos escritos en lengua francesa. Sin embargo, hacia el final, tanto en el primer caso como en el segundo, la culminación de un texto que hasta el momento se avocaba mayormente a la utilización de un estilo refinado, da un giro y adquiere una tonalidad semejante a la de un reportaje, con menciones históricas sobre la presencia de las flores y su propagación por los territorios de Francia y Chile durante los siglos XVII y XVIII, o críticas acerca del ambiente teatral francés del momento: "La obra teatral debe ser comprendida, completamente gozada por la muchedumbre. No debe ser la obra de un grupo de iniciados, debe ser la obra de todos" (Ambrogi, 1963, p. 229).

Si se analizan los textos más de cerca, queda manifiesto cómo, en realidad, lo que Ambrogi escribe se adapta y rehúye al mismo tiempo de las categorizaciones totales que se han explicado sobre el modernismo y la crónica en Latinoamérica. Muchas de las reflexiones hechas por el autor incluso se sitúan en un lugar de enunciación personal, casi como si se estuviera ante la escritura de un diario de viajes. En algunos casos, los textos presentes en Marginales de la Vida (1963) responden directamente a la experiencia del autor como personaje cultural en una región de principios de siglo que, claramente, estaba siendo impactada por las ideas y el pensamiento provenientes de otras latitudes, cuyos resultados se aprecian en reinterpretaciones como las practicadas tan desenfadadamente por Ambrogi en el texto estudiado. El diálogo entre su memoria y la de otros de sus contemporáneos empieza a tonarse, en estos casos, en un lugar de discusión para visiones que exceden lo artístico, lo literario y lo imaginativo, como se observa en el testimonio que escribe sobre su visita a la capital de Costa Rica:

Cuando (va a hacer de ello apenas un año) la suerte...y la aventura, me llevaron de nuevo a Costa Rica, en ese inolvidable San José, que encontré transformado en doce años de ausencia, supe, con pesar, que aquella amistad fraternal había concluido. (Ambrogi, 1963, p. 41)

Esta cita se enmarca en uno de sus textos más extensos, titulado Con don Ricardo Jiménez (1963), en donde se habla de las relaciones entre este personaje de la política costarricense y su homólogo Alfredo González Víquez, así como del estado de la creciente urbe josefina y parte de su idiosincrasia. Es también particular notar el análisis que Ambrogi hace de la escena política en Costa Rica, el cual se 
lleva a cabo bajo un registro igualmente híbrido que intercala tonalidades narrativas con hechos que bien podrían desprenderse de las noticias publicadas en los periódicos de la época, siempre teniendo en cuenta que lo que se dice ha tomado como base acciones concretas de las cuales el autor fue testigo:

Todo el mundo acude al congreso, y llena sus insuficientes galerías, obstruye sus partes, invade el enlosado -"Hoy habla Ricardo"- "Va a hablar Ricardo"-. La buena nueva se propala con rapidez, y ese momento parlamentario, es de lo más trascendental y característico de la vida política costarricense. (Ambrogi, 1963, p. 43)

Como se puede ver, tal y como lo afirmó Edberg (1965), este y una buena parte de los textos compilados en Marginales de la vida (1963) poseen un sustento que evidentemente proviene de la experiencia directa de quien los cuenta. Lo anterior, de hecho, también da la ocasión de señalar la ausencia que hay en el libro de algún fragmento pueda tildarse de ficticio o inventivo en su acepción más general, algo que se observa de entrada, al leer algunos de los títulos que se despliegan en el índice, como El General Mansilla, Ante los escombros del Nacional, La floresta de Leones, o La muerte de Andrés Theurriet. Si bien los textos reunidos no siguen un orden determinado ni un hilo temático único, se puede afirmar que cada uno ofrece un registro lineal de personajes, lugares, acontecimientos o situaciones estrechamente relacionadas con un espacio y unas circunstancias.

Dicho aspecto deja claro que resultaría forzado encasillar la obra de Ambrogi exclusivamente dentro de la estética modernista latinoamericana, pues como se puede ver, los textos de Marginales de la vida (1963) solo parecen ser modernistas en tanto han sido escritos siguiendo un alto rigor estilístico y verbal; así, los rasgos creacionistas del modernismo no podrían encontrarse en la obra del autor salvadoreño precisamente porque su escritura no busca huir absolutamente de lo que se veía y se vivía en su momento histórico. Su visión de lo que escribe más bien parece estar comprometida con el acontecer social y político tanto dentro como fuera de su país, un punto que también se puede apreciar en los textos en los cuales Ambrogi se explaya en sus apreciaciones acerca de determinados acontecimientos de diversa índole, por ejemplo, en el que lleva por nombre La paz Ruso-japonesa en El Salvador (1963), donde reflexiona acerca de los intereses ideológicos que se desarrollan entre unas y otras naciones, así como de las consecuencias que dichos pormenores llegan a tener a largo plazo para su propio país:

Ahora, una noticia que el Diario del Salvador (sic) consigna en su edición de ayer tarde, viene a probar con datos aún más fehacientes, el hondo interés que nos tomamos por la suerte de nuestras grandes y buenas amigas las Potencias. (Ambrogi, 1963, p. 370) 
Se trata, nuevamente, de un repaso por la historia que se estaba escribiendo en el momento en el que Ambrogi también llevaba a cabo su producción literaria. En este sentido, si la lectura del texto continúa, resulta sencillo notar que los acuerdos a los que los rusos y los japoneses han llegado para poner fin a la guerra citada funcionan en realidad como es una excusa para que el autor ironice acerca del alcance y la difusión que un acontecimiento de tal envergadura puede alcanzar en un territorio como el salvadoreño, donde mucho del saber difundido no va más allá de un excesivo deslumbramiento por lo europeo, paradójicamente practicado por él en más de una oportunidad.

Esto a su vez da la ocasión de coquetear con algo en lo que Ambrogi no incursiona, pero al parecer conoce: la posibilidad de inventar noticias para vender diarios y obtener publicidad, aprovechándose, entre otras cosas, de dicho deslumbramiento, de la lejanía geográfica y temporal de algunos hechos, y del desconocimiento generalizado por parte de la población, aspectos que el autor solamente menciona con el mismo guiño de ironía, sin llegar a incursionar en ellos. No sería aventurado afirmar que Ambrogi obvia dicha estrategia porque esta configuración de lo literario se encuentra alejada de lo que realmente desea plasmar. Lo que interesa en su escritura es precisamente lo contrario, es decir, dejar de lado la naturaleza inventiva de la literatura y (en un giro parecido a lo argumentado por Pérez-Grande (2008) sobre la página en blanco y el desafío compartido entre cronistas y escritores de ficción) utilizar los mismo medios del discurso literario para implementarlos como un mecanismo de localización de lo que se dice dentro del marco de lo cotidiano, de lo circunstancial, y bajo la perspectiva de lo que se experimentaba desde una modesta nación del istmo.

Lo anterior justificaría en parte la clasificación que la historiografía tradicional ha hecho al colocar la producción de Ambrogi dentro de los esquemas del costumbrismo y del localismo salvadoreños del siglo XX, pues estos resultan ser lugares idóneos para ubicar textualidades que, como las de Ambrogi, se acercan de forma clara al realismo, al menos en las dimensiones temática y referencial. Aún con esto, dicha clasificación podría ser puesta en tela de duda a partir de una discusión que problematice la injerencia que otros elementos, como el estilo y los antecedentes tanto contextuales como estéticos utilizados por el autor, tienen en dichos modelos de categorización, ya que en muchos casos lo que hacen de forma enfática es violentarlos y generar la aparición de otros puntos de vista que no necesariamente son encasillados o encasillables.

Otras apreciaciones pertinentes para el caso en análisis, como la toma del espacio cotidiano mediante la crónica periodística argumentada por Reguillo (2000), también se observan en los textos presentes en el libro de Ambrogi, especialmente por medio de menciones a personas y sujetos que entran en contacto entre sí con 
la ciudad como telón de fondo. Este formato, más cercano a lo que algunos de los autores repasados denominan como cuadro de costumbres, se construye con la intensión de testimoniar un instante en el transcurso habitual del tiempo, sin que exista la necesidad de organizar dicho instante a partir de trucos argumentales de algún tipo. Uno de estos casos se aprecia en la crónica titulada Un japonés en El Salvador (1963), cuyo contenido retrata, precisamente, la imagen de un oriental paseándose por la capital salvadoreña:

Chiquitín, vestido de marinero, con sus calzones de campana meciendo exageradamente los brazos al caminar, con esa ondulación del paso que da el hábito de la vida en un barco, llamaba la atención de los pacíficos habitantes de San Salvador. (Ambrogi, 1963, p. 381)

Este texto en particular ejemplifica muchos de los rasgos discutidos hasta el momento en la obra de Arturo Ambrogi. Por un lado, se señala la imagen de un oriental con asombro y extrañeza, casi como si se tratara de un recordatorio sobre la perspectiva que se tenía acerca del exotismo de los crisantemos, provenientes también de Japón; por el otro, el texto se aparta de cualquier apreciación fantasiosa o espiritual y, en cambio, se toma la tarea de retratar el modo en el cual dicho personaje se inserta y se percibe en medio del ambiente urbano y social salvadoreño. Dicha conjugación parece reunir las cualidades que se han señalado acerca de Marginales de la vida (1963), a saber, la apropiación (y por qué no, reinvención) de códigos de distinta naturaleza que se utilizan para dar testimonio de acciones emplazadas no en el imaginario promulgado por los modernistas, sino en la experiencia de quien habla de ellas.

El recurso anterior puede igualmente relacionarse con lo señalado por Reguillo (2000) sobre la posibilidad de la crónica de yuxtaponer versiones y anécdotas que se acercan al territorio de lo propio, lo cual de hecho, también fundamentaría la inclusión de la obra de Ambrogi dentro de la estética costumbrista. Al fin y al cabo, lo que sus textos proponen es la localización de lo escrito dentro de un marco espacio-temporal claramente identificado. Si el modernismo en su aplicación más rigurosa buscaba elevarse por encima de lo terrenal y crear nuevas formas a partir de la nada absoluta, los textos en Marginales de la vida (1963) desvían dicha concepción y, por el contrario, se encargan de emplazar y territorializar el discurso que se enuncia. El título mismo del libro podría plantearse como un juego entre la rigurosidad moderna que Ambrogi practica en su escritura, y el uso de recursos o espacios que, en principio, no coinciden de modo directo con dicha rigurosidad porque son marginales.

En general, se ha podido apreciar lo expuesto al inicio acerca de la oscilación presente en la obra de Arturo Ambrogi, particularmente respecto de Marginales de la vida (1963), los textos de este libro se resisten en muchas de sus características a 
encasillarse completamente dentro de una única corriente estética y, en cambio, conforman un sistema de escritura en el cual entran en contacto diversas formas de "literarizar" el entorno y las percepciones que se tenían sobre este. En lo relativo a la tesis, la oscilación citada al inicio se puede demostrar en el análisis de los textos expuestos en las páginas anteriores, en donde, de modo indiferente, la voz de quien escribe toma recursos de ambos polos para concretar lo que dice, en una estrategia similar a la argumentada por Pérez-Grande (2008) sobre el individuo que escribe y que utiliza los recursos que tenga a mano para llevar a buen término su empresa.

Queda por estudiar más a fondo las posibles consecuencias que la tesis planteada en este artículo pueda llegar a tener en la compresión de visiones como la establecida por Reguillo (2000) acerca de la crónica y la prosa modernista como formas que surgieron bajo principios opuestos durante los primeros años del siglo XX. De igual modo, un análisis de la totalidad de la obra del autor salvadoreño podría arrojar más luz acerca de su apropiación de la estética modernista y del oficio periodístico, los cuales, como se ha podido observar, se entrelazan en la mayoría de sus textos sin llegar a dibujar un límite que las separe de forma completa. La compilación estudiada en este trabajo se vincula con la interpretación citada por Meyer-Minnemann (1987) acerca de la complejidad de analizar la prosa escrita durante y después del modernismo y, si ese hecho se hace palpable en el resto de su literatura, la obra de Ambrogi podría servir como una demostración aún más sólida de este último punto, el cual, en última instancia, debería ser tomado en consideración al momento de ahondar en los esquemas de clasificación literaria no sólo de este periodo, sino de cualquier otro.

\section{Referencias}

Acereda, A. (2001). Modernismo y modernidad: Deslindes de Una Poética Dariana. Chasqui, 2, 20-34.

Ambrogi, A. (1963). Marginales de la vida. $2^{\mathrm{a}}$ ed. San Salvador: Ministerio de Educación.

Bradford-Burns, E. (1985). The Intellectual Infrastructure of Modernization in El Salvador, 1870-1900. The Americas, 41, 57-82.

Canales, T. (1978). Arturo Ambrogi: análisis de su obra. Anuarios de Estudios Centroamericanos, 4, 247-277.

Edberg, G. J. (1965). Review of Marginales de la vida. Hispania, 48, 185-186.

Meyer-Minnemann, K. (1987). Lo moderno del modernismo. Ibero-amerikanisches Archiv, Neue Folge, 13, 77-91. 
Pérez-Grande, H. (2008). Relaciones sospechosas: literatura y periodismo. Caravelle, 90, 203-205.

Reguillo, R. (2000). Textos fronterizos. La crónica: una escritura a la intemperie. Guaraguao, 11, 20-29. 